\title{
Neuronic autophagy contributes to p-connexin 43 degradation in hippocampal astrocytes following traumatic brain injury in rats
}

\author{
LI QIAN SUN ${ }^{1}$, JUN LING GAO ${ }^{2}$, YING CUI ${ }^{2}$, MAN MAN ZHAO $^{2}$, XIAO BIN JING ${ }^{3}$, \\ RAN LI $^{2}$, YAN XIA TIAN ${ }^{2}$, JIAN ZHONG CUI ${ }^{4}$ and ZHONG-XUE WU ${ }^{1}$ \\ ${ }^{1}$ Department of Interventional Neuroradiology, Beijing Neurosurgical Institute and Beijing Tiantan Hospital, \\ Capital Medical University, Beijing 100050; ${ }^{2}$ Department of Histology and Embryology, School of \\ Basic Medical Science, Hebei United University; ${ }^{3}$ Department of Ophthalmology, Tangshan Ophthalmology Hospital; \\ ${ }^{4}$ Department of Neurosurgery, Tangshan Gongren Hospital, Tangshan, Hebei 063000, P.R. China
}

Received April 7, 2014; Accepted August 5, 2014

DOI: $10.3892 / \mathrm{mmr} .2015 .3264$

\begin{abstract}
Connexins, gap junction proteins, have short half-lives of only a few hours; therefore, degradation of these proteins can rapidly modulate their function. Autophagy is a type of degradation pathway that has been implicated in several diseases and was reported to be induced following traumatic brain injury (TBI). The aim of the present study was to investigate the involvement of neuronic autophagy in proteolysis of phosphorylated connexin 43 (p-Cx43) in hippocampal astrocytes following TBI in rats. Western blot analysis and immunofluorescence showed a TBI-induced increase in levels of astrocytic $\mathrm{p}-\mathrm{Cx} 43$ following treatment with 3-methyladenine, an inhibitor of autophagy, in the hippocampus. Internalized gap junctions were observed in the neuronic cytoplasm using transmission electron microscopy. These results demonstrated that neuronic autophagy may regulate cellular levels of $\mathrm{p}-\mathrm{Cx} 43$ in hippocampal astrocytes following TBI. This therefore indicated that the persistence of $\mathrm{p}-\mathrm{Cx} 43$ accumulation was due to insufficient degradation capacity of constitutive autophagy.
\end{abstract}

\section{Introduction}

Cellular proteins are continually renewed by synthesis and degradation. Macroautophagy, a process by which cells autodigest parts of their cytoplasm, is a fundamental cellular mechanism of protein degradation (1). Autophagy is an evolutionarily conserved pathway that leads to stress-induced degradation of intracellular proteins and organelles (2). This type of degradation has been reported to be mediated

Correspondence to: Dr Zhong-Xue Wu, Department of Interventional Neuroradiology, Beijing Neurosurgical Institute and Beijing Tiantan Hospital, Capital Medical University, 6 Tiantan Xili, Chongwen, Beijing 100050, P.R. China

E-mail: zhongxuewu@yeah.net

Key words: autophagy, gap junction, microtubule-associated protein 1 light chain 3, p-connexin-43, traumatic brain injury by interactions with microtubule-associated protein 1 light chain 3 (LC3), a mammalian homologue of autophagy-related gene 8 , which was demonstrated to proceed via recruitment to the phagophore/isolation membrane, where it remained associated with the completed autophagosome (3).

The present study investigated the involvement of autophagy in the degradation of gap junction proteins following traumatic brain injury (TBI). Gap junctions are components of the plasma membrane that contain clusters of oligomeric assemblies of proteins called connexins (Cxs), which form channels to allow the passage of ions and small molecules between adjacent cells. Previous studies have shown that several Cxs are expressed by numerous types of cells in the central nervous system (4). Cx43 was shown to be expressed exclusively in astrocytes (5), demonstrated using immunogold labeling for $\mathrm{Cx} 43$ (6). Phosphorylated Cx43 (p-Cx43), via the extracellular regulated protein kinase signaling pathway, reduces intercellular permeability via gap junctions, correlating with tissue homeostasis (7). Using HeLa and normal rat kidney epithelial cell models, Lichtenstein et al (8) reported that autophagy-regulated connexin degradation may be induced by starvation. However, the mechanism of neuronic autophagy, which contributed to astrocytic $\mathrm{p}-\mathrm{Cx} 43$ degradation in the hippocampus following TBI in vivo, remains to be elucidated. In the present study, the involvement of autophagy in the degradation of $\mathrm{p}-\mathrm{Cx} 43$ was examined in the rat hippocampus following experimental TBI.

\section{Materials and methods}

Animals and TBI model. The study was approved by the ethics committee of the Hebei United University (Tangshan, China). All experimental procedures were performed in accordance with the guidelines of the Chinese Council on Animal Protection and were approved by Hebei United University Committee for the Use of Animals in Research (Tangshan, China). A total of 60 male Sprague-Dawley rats (age, 12-16 weeks; weight, 350-375 g; Vital River Laboratory Animal Technology Co., Ltd., Beijing, China) were used in the present study. All animals were housed using the standard 12-h light/dark cycle 
and access to water and food ad libitum prior to and following surgery or sham operation. The rat model of TBI was induced using a modified weight-drop device, as described previously by Marmarou et al (9). In brief, rats were anaesthetized using sodium pentobarbital (Nembutal, $60 \mathrm{mg} / \mathrm{kg}$ ). A midline incision was made that exposed the skull between the bregma and lambda suture lines, and a steel disc $(10 \mathrm{~mm}$ in diameter and $3 \mathrm{~mm}$ in thickness; Tangshan Railway Vehicle Co., Ltd., Tangshan, China) was then adhered to the skull using acrylic polymers. Animals were subsequently placed onto a foam mattress (Tangshan Railway Vehicle Co., Ltd.) positioned underneath a weight-drop device where a 450-g weight fell freely through a vertical tube from $1.5 \mathrm{~m}$ onto the steel disc. Sham-operated animals underwent an identical surgical procedure without weight-drop impact. Following surgery, rats were housed in individual cages and placed on heat pads $\left(37^{\circ} \mathrm{C}\right)$ for $24 \mathrm{~h}$ to maintain a normal body temperature during the recovery period.

Group determination and administration of drugs. The rats were randomly divided into three groups: Sham, TBI, and TBI treated with 3-methyladenine (3-MA; Sigma-Aldrich, St. Louis, MO, USA) ( $n=5$ for each group). The rats were sacrificed at time-points of 3, 6, 24 and $48 \mathrm{~h}$ following TBI. 1 1 1 3-MA (sc-205596, 400 nmol; Santa Cruz Biotechnology, Santa Cruz, CA, USA) was administered through right ventricle injection $10 \mathrm{~min}$ prior to the induction of TBI. For intracerebroventricular injections, animals were fixed in a stereotaxic apparatus (RWD68025; RWD Life Science Co., Ltd., Shenzhen, China), a midline incision was made in the skin, followed by the induction of a small hole in the cranial region. A Hamilton syringe (RWD68025; RWD Life Science Co., Ltd.) was used to inject 3-MA or $1 \mu \mathrm{l}$ saline into the right cerebral ventricle according to the following coordinates: Bregma, advanced placement $-0.8 \mathrm{~mm}$, right $+1.6 \mathrm{~mm}$ (midline) and deep $3.4 \mathrm{~mm}$ from dura (10). Sham-operated rats received intracerebroventricular injection of $1 \mu \mathrm{l}$ normal saline. Hippocampal tissue (the entire ipsilateral hippocampus, including the impact site and surroundings) from each injured rat was dissected and used for numerous assays.

Western blot analysis. Western blot analysis was performed as previously described (11). In brief, rats were deeply anesthetized and underwent intracardiac perfusion with $0.1 \mathrm{~mol} / 1$ phosphate-buffered saline (PBS; pH 7.4; Wuhan Boster Biological Engineering Co., Ltd., Wuhan, China). The hippocampus was rapidly isolated; total proteins were extracted and protein concentration was determined using the bicinchoninic acid reagent (Solarbio, Beijing, China) method. Samples were subjected to SDS-PAGE. Separated proteins on the gel were transferred onto polyvinylidene fluoride membranes (Roche Diagnostics, Mannheim, Germany). Blots were blocked using 5\% fat-free milk powder (Wuhan Boster Biological Engineering Co., Ltd.,) for $1 \mathrm{~h}$ at room temperature. Subsequently, blots were incubated overnight at $4^{\circ} \mathrm{C}$ with indicated primary antibodies, including rabbit anti-p-Cx43 polyclonal antibodies, rabbit anti-LC3 polyclonal antibody and mouse anti- $\beta$-actin monoclonal antibody (dilution, 1:500; Santa Cruz Biotechnology; Santa Cruz, CA, USA). The blots were then incubated with horseradish peroxidase-conjugated anti-rabbit and anti-mouse immunoglobulin G (dilution, 1:5,000; Cell Signaling Technology, Danvers, MA, USA) for $2 \mathrm{~h}$ at room temperature. Following incubation with titrated secondary antibodies, the immunoblot on the polyvinylidene fluoride membrane was visible once developed using an enhanced chemiluminescence detection system (ChemiDoc XRS; Bio-Rad Laboratories, Hercules, CA, USA), and the densitometric signals were quantified using imaging software (Image Lab 4.1; Bio Rad Laboratories). Immunoreactive bands of the protein expression levels were normalized to the intensity of corresponding $\beta$-actin bands. The western blot results were analyzed Image J software (Bethesda, MD, USA).

Immunofluorescence analyses. The brain tissues were fixed in 4\% paraformaldehyde (Wuhan Boster Biological Engineering Co., Ltd.) for $24 \mathrm{~h}$, followed by immersion in $30 \%$ sucrose solution until sinking to the bottom. Sections were made $200 \mu \mathrm{m}$ apart from the anterior to posterior hippocampus (bregma -1.90 to $-3.00 \mathrm{~mm}$ ) of the TBI animals and then embedded in optimal cutting temperature compound (Beijing Solarbio Science \& Technology Co., Ltd., Beijing, China). $15-\mu \mathrm{m}$ frozen sections were sliced with a cryostat (CM 1950, Heidelberger, Germany) treated with $0.4 \%$ Triton-100 for $10 \mathrm{~min}$ and then blocked in normal donkey serum for 1 h (both Beijing Solarbio Science \& Technology Co., Ltd.). For triple labeling, frozen sections were incubated with a mixture of goat anti-p-Cx43 polyclonal antibody, rabbit anti-LC3 polyclonal antibody and mouse anti-glial fibrillary acidic protein (GFAP) monoclonal antibody (dilution, 1:100; Santa Cruz Biotechnology, Santa Cruz, CA, USA) overnight at $4^{\circ} \mathrm{C}$. The next day, the sections were incubated with Alexa Fluor 647-conjugated, 594-conjugated and 488-conjugated secondary antibodies (dilution, 1:500; Invitrogen, Carlsbad, CA, USA) against the appropriate species for $2 \mathrm{~h}$ at $37^{\circ} \mathrm{C}$ in the dark. Sections were mounted with DAPI (Vector Laboratories, Burlingame, CA, USA). Images were captured using a laser scanning confocal microscope (Olympus FV1000; Olympus, Tokyo, Japan). Primary antibodies were replaced with PBS in the negative control group.

Electron microscopy. Freshly dissected tissues were fixed in $3 \%$ glutaraldehyde and $1 \%$ osmic acid for $2 \mathrm{~h}$, followed by propionaldehyde (Beijing Zhongshan Jinqiao Biology \& Technology Co., Ltd., Beijing, China) dehydration and epoxy resin embedding. Tissue was sliced using a cryostat and stained using 2\% uranyl acetate and lead citrate (Beijing Zhongshan Jinqiao Biology \& Technology Co., Ltd.). Images were observed and recorded using transmission electron microscopy (Hitachi, Tokyo, Japan).

Statistical analysis. All experiments were repeated three times and similar results were obtained. Statistical analysis was performed using the SPSS 16.0 statistics software (IBM, Armonk, NY, USA). Data are expressed as the mean \pm standard deviation. Statistical analysis was performed using analysis of variance, followed by the Dunnett's post-hoc test. $\mathrm{P}<0.05$ was considered to indicate a statistically significant difference between values. 
A

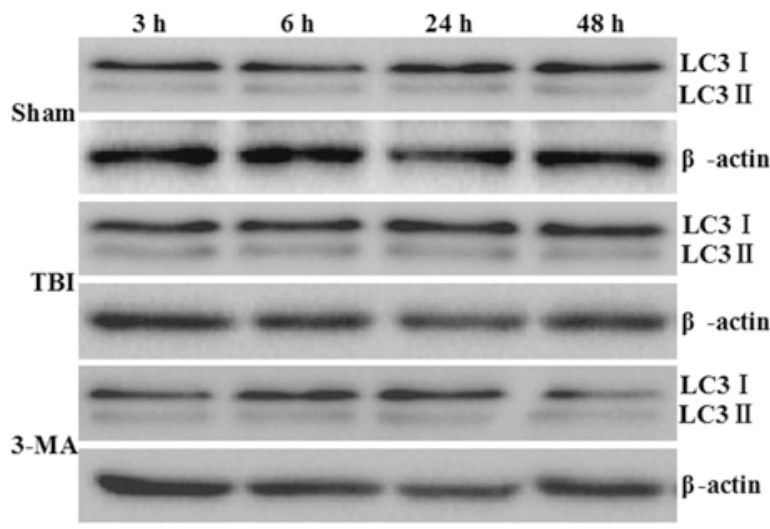

B

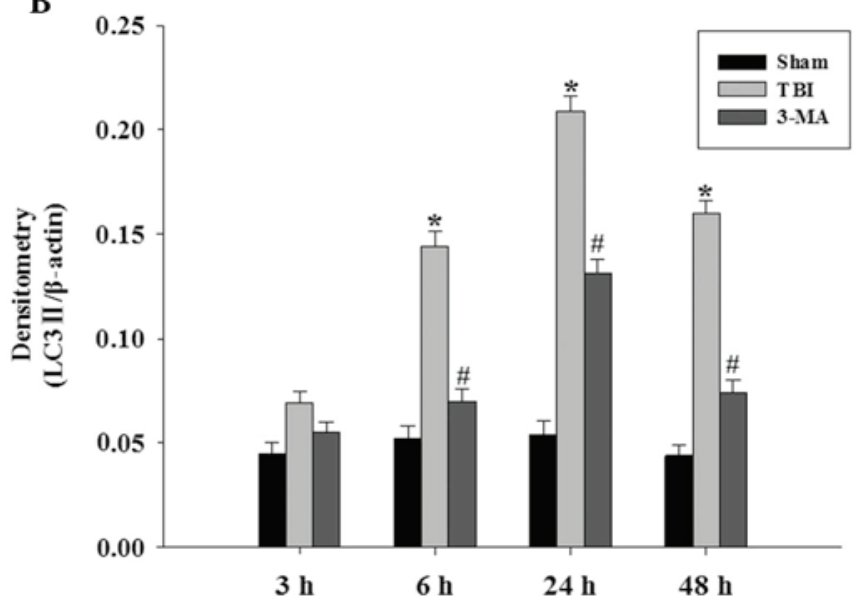

Figure 1. Pretreatment with 3-MA prevents TBI-induced LC3-II activation. (A) Western blot analysis of LC3-II bands in the hippocampus and (B) densitometric analysis of LC3-II bands corresponding to $\beta$-actin. Bars represent the mean \pm standard deviation $(\mathrm{n}=5)$. 3-MA, 3-methyladenine; TBI, traumatic brain injury; LC3, microtubule-associated protein 1 light chain 3.

\section{Results}

General. No significant differences were observed in body weight or temperature between the TBI and sham-injured groups. Furthermore, no differences were found in injury levels among the 3-, 6-, 24-, or 48-h TBI groups.

3-MA treatment depresses LC3-II protein levels. LC3-II protein levels were analyzed using western blot analysis (Fig. 1A). LC3-II protein was identified at low levels in the hippocampus of rats in the sham group. Activation of LC3-II in the hippocampus was significantly increased at $6 \mathrm{~h}$ following injury, peaked at $24 \mathrm{~h}$, and by $48 \mathrm{~h}$ activation of LC3-II had slightly decreased but was still significant ( $\mathrm{P}<0.05$ vs. sham group). The results shown in Fig 1B demonstrated that 3-MA pretreatment significantly inhibited the upregulation of LC3-II protein levels compared with those of the TBI groups at 6, 24 and $48 \mathrm{~h}(\mathrm{P}<0.05$ vs. TBI group).

p-CX43 is increased following TBI in the rat hippocampus. Protein levels of p-Cx43 were determined in order to observe altered $\mathrm{p}-\mathrm{Cx} 43$ activity following TBI and confirm the ability of 3-MA to regulate p-Cx43 (Fig. 2A). Levels of p-Cx43 protein in the hippocampus were significantly upregulated at

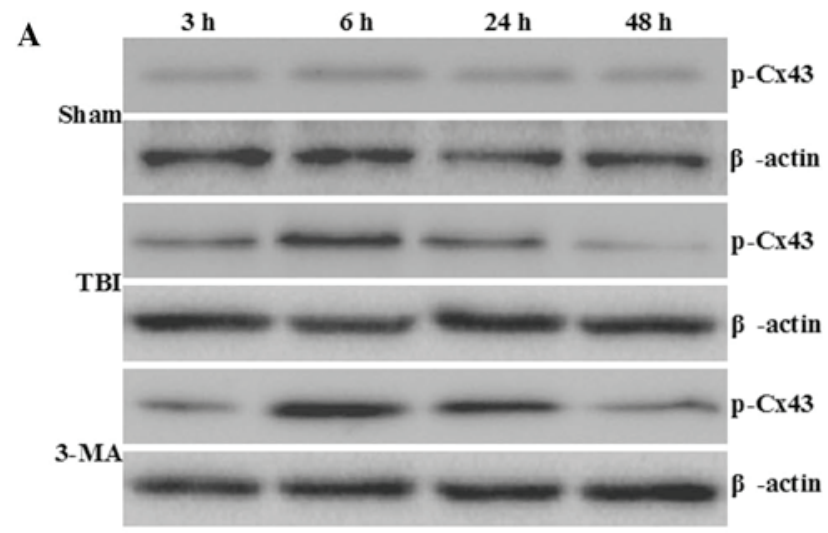

B

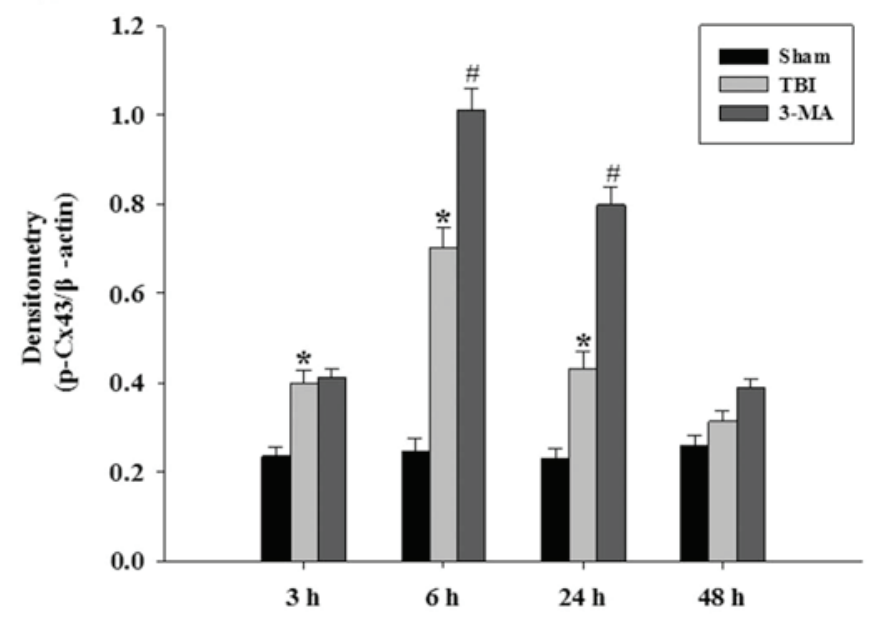

Figure 2. Pretreatment with 3-MA increases TBI-induced p-Cx43 activation. Protein levels of $\mathrm{p}-\mathrm{Cx} 43$ in the hippocampus were detected using (A) western blot analysis and (B) densitometric analysis of p-Cx43 with bands corresponding to $\beta$-actin. Data are expressed as the mean \pm standard deviation $(n=5)$. 3-MA, 3-methyladenine; TBI, traumatic brain injury; p-Cx43, phosphorlyated connexin 43 .

$3 \mathrm{~h}$ following TBI, which persisted at high levels until $24 \mathrm{~h}$ following injury ( $\mathrm{P}<0.05$ vs. sham group). As demonstrated in Fig. 2B, pretreatment with 3-MA significantly increased the relative protein abundance of $\mathrm{p}-\mathrm{Cx} 43$ in the hippocampus at 6 and $24 \mathrm{~h}$ following TBI ( $\mathrm{P}<0.05$ vs. TBI group).

Neuronic autophagy regulates immunofluorescence intensity of astrocytic p-Cx43 in the hippocampus following TBI. An overlap was observed between GFAP and p-Cx43 in hippocampal astrocytes, indicating that $\mathrm{p}-\mathrm{Cx} 43$ strongly colocalized with the hippocampal astrocytes (Fig. 3; arrows). DAPI analysis revealed that LC3 specifically colocalized within neurons of the hippocampus. The immunofluorescence intensity of LC3 and $\mathrm{p}-\mathrm{Cx} 43$ in the hippocampus was significantly increased at $24 \mathrm{~h}$ following TBI. It was also found that pretreatment with the autophagy inhibitor 3-MA further enhanced the TBI-induced increase in p-Cx43 immunofluorescence intensity in hippocampal astrocytes.

Internalized gap junctions are present in lysosomes in the hippocampal neuron. Connexin and gap junction degradation was further analyzed by examining the ultrastructure of rat hippocampi using transmission electron microscopy $24 \mathrm{~h}$ 

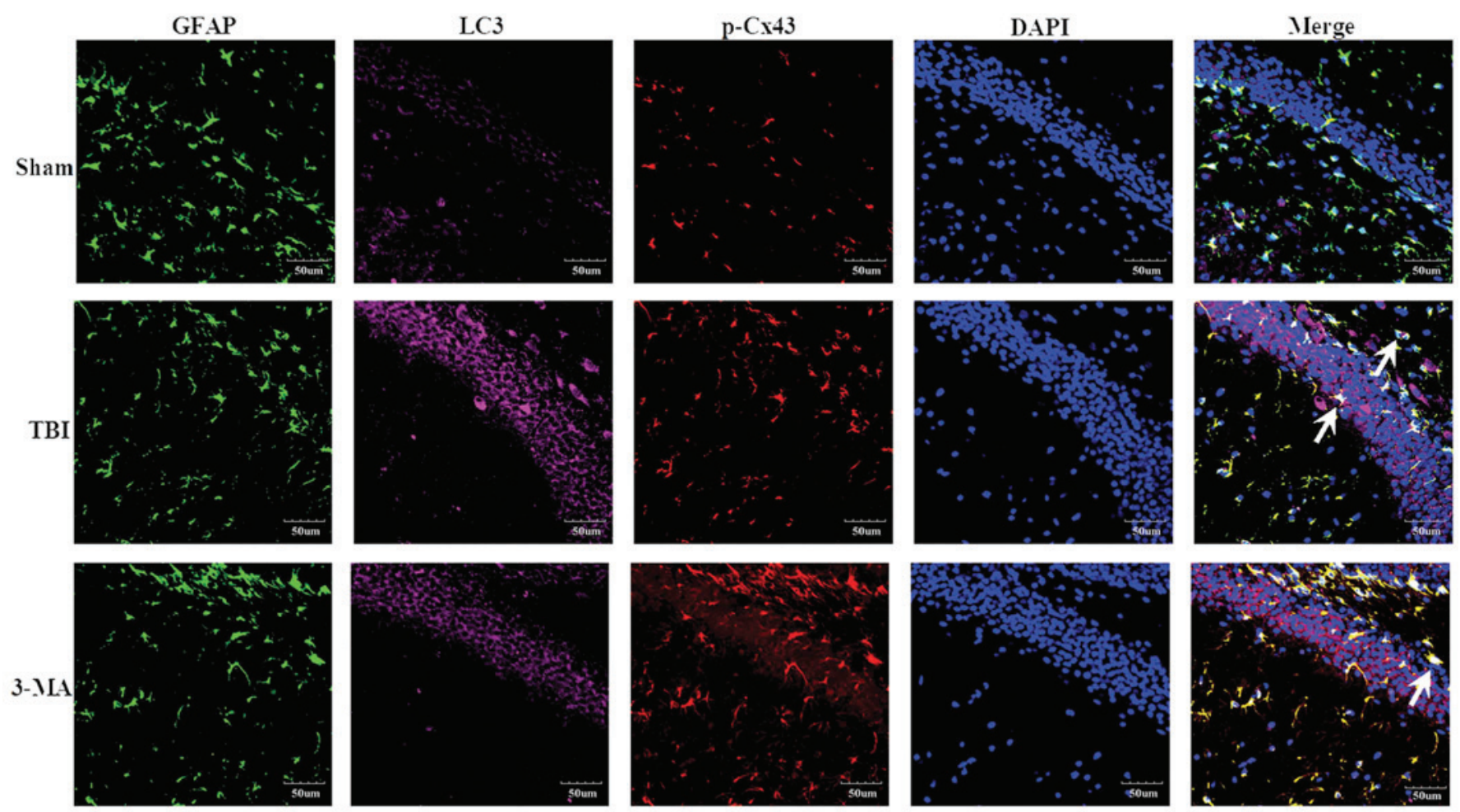

Figure 3. 3-MA treatment increases the immunoreactivity of p-Cx43 in hippocampal astrocytes. Confocal microscopy images show p-Cx43 (red), GFAP (green) and LC3 (purple) in the hippocampus under sham conditions, $24 \mathrm{~h}$ following TBI and 3-MA pretreatment followed by TBI. The merge panel demonstrates the colocalization of the three signals. Blue represents DAPI staining of nuclei. 3-MA, 3-methyladenine; TBI, traumatic brain injury; p-Cx43, phosphorlyated connexin 43; glial fibrillary acidic protein; LC3, microtubule-associated protein 1 light chain 3; GFAP, glial fibrillary acidic protein. Magnification, $\mathrm{x} 400$.

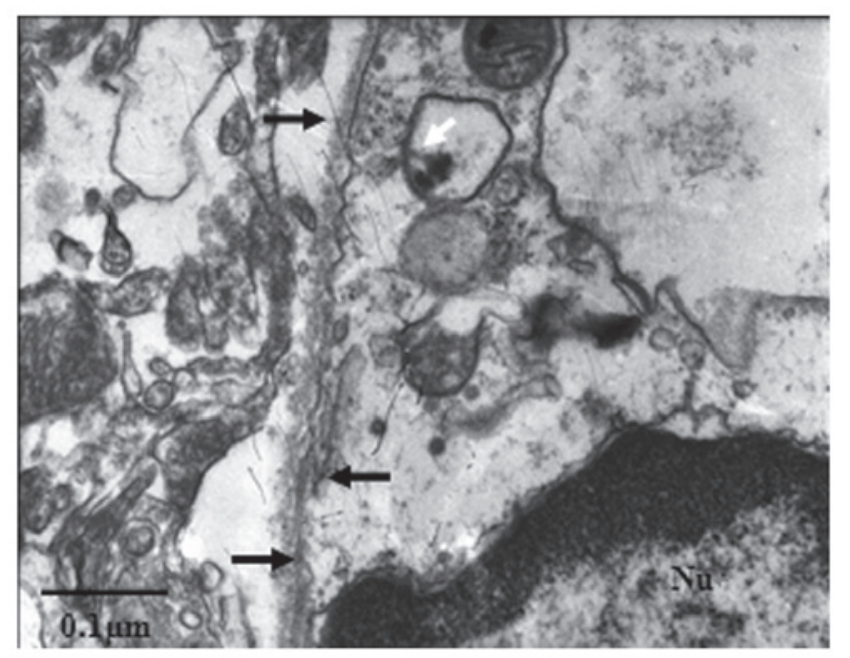

Figure 4. Internalized gap junctions were observed in lysosomes in hippocampal neurons at $24 \mathrm{~h}$ following TBI. Electron micrograph showing gap junctions (black arrows) and secondary lysosome (white arrow). Nu, nucleus; TBI, traumatic brain injury. Magnification, x30,000.

following TBI. Gap junction structures were found between astrocytes and neurons (Fig. 4; black arrows). Profiles of the internalized gap junctions were observed in the neuronic cytoplasm. Certain profiles of partially disrupted internalized gap junctions were observed inside membranous structures; these structures are likely to represent secondary lysosomes (Fig. 4; white arrow).

\section{Discussion}

Autophagy is a proteolytic process which, under normal conditions, demonstrates low levels of activity; however, under pathological conditions, the activity of this pathway is significantly altered (12). The ability of cells to increase their autophagic proteolytic activity enables them to adapt to changes in their environment, such as TBI (13). The results of the present study showed that protein levels of p-Cx43 in the hippocampus were significantly upregulated at 3,6 and $24 \mathrm{~h}$ following TBI, whereas other studies indicated that no significant changes in the total amount of $\mathrm{Cx} 43$ in the hippocampus were present following injury (5). The results of the present study suggested that $\mathrm{p}-\mathrm{Cx} 43$ in the hippocampus is associated with molecular sequelae of TBI. Previous studies have demonstrated using biochemical and colocalization studies that astrocytic $\mathrm{p}-\mathrm{Cx} 43$ regulated neuronal autophagy in the hippocampus following TBI in rats (14). Autophagy was found to be involved in TBI-induced cell membrane breakdown, cell loss as well as motor and cognitive outcome deficits (15). However, studies have revealed that reduction of cell-cell permeability through gap junctions may lead to partial pathological hippocampal dysfunction, including post-traumatic amnesia and post-traumatic stress disorders $(16,17)$. This therefore highlights the importance of elucidating the cellular mechanisms of the involvement of neuronic autophagy in astrocytic connexin degradation.

Ultrastructural studies in rat liver, incisor and fetal epidermis (18) as well as equine hoof wall (19) have revealed 
the internalization of gap junctions enclosed within double membrane structures, therefore indicating the involvement of autophagic activity in the degradation of gap junctions or connexins. Gap junctions between neurons and astrocytes function as an interdependent network with bidirectional communication (20). In the present study, autophagic double membrane structures were observed at the 1-h time-point post-TBI in neurons, and three days later in astrocytes. Western blot analysis revealed that 3-MA significantly increased protein levels of $\mathrm{p}-\mathrm{Cx} 43$ following TBI-induction in the hippocampus, therefore indicating the involvement of autophagy in the TBI-induced degradation of p-Cx43 in the hippocampus. Immunofluorescence assays also demonstrated increased immunofluorescence activity of astrocytic $\mathrm{p}-\mathrm{Cx} 43$ in the hippocampus following treatment with 3-MA compared with that of the TBI group without 3-MA-treatment. This therefore demonstrated the involvement of neuronic autophagy in the degradation of astrocytic p-Cx43 in the hippocampus. Internalized gap junctions surrounded by lysosomal structures were observed in hippocampal neurons using ultrastructural studies, implicating the involvement of neuronic autophagy in the degradation of gap junction plaques, which may be formed of astrocytic connexin. It was therefore suggested that astrocytic $\mathrm{p}-\mathrm{Cx} 43$ targeted for subsequent degradation and gap junction plaques between astrocyte and neuron are sequestered by an LC3-containing membrane following stimulation of neuronic autophagy by TBI in the hippocampus.

In conclusion, the present study suggested that autophagy in neurons was involved in the degradation pathway for $\mathrm{p}-\mathrm{Cx} 43$ in hippocampal astrocytes following TBI.

\section{Acknowledgements}

The present study was supported by a grant from the Natural Science Foundation of Hebei Province (no. H2012401071).

\section{References}

1. Reggiori F and Klionsky DJ: Autophagosomes: biogenesis from scratch? Curr Opin Cell Biol 17: 415-422, 2005.

2. Pozuelo-Rubio M: Regulation of autophagic activity by 14-3-3६ proteins associated with class III phosphatidylinositol-3-kinase. Cell Death Differ 18: 479-492, 2011.

3. Zheng YT, Shahnazari S, Brech A, Lamark T, Johansen T and Brumell JH: The adaptor protein p62/SQSTM1 targets invading bacteria to the autophagy pathway. J Immunol 183: 5909-5916, 2009.
4. De Bock M, Kerrebrouck M, Wang N and Leybaert L. Neurological manifestations of oculodentodigital dysplasia: a Cx43 channelopathy of the central nervous system? Front Pharmacol 4:120, 2013.

5. De Maio A, Vega VL and Contreras JE: Gap junctions, homeostasis, and injury. J Cell Physiol 191: 269-82, 2002.

6. Rash JE, Yasumura T, Dudek FE and Nagy JI: Cell-specific expression of connexins and evidence of restricted gap junctional coupling between glial cells and between neurons. J Neurosci 21: 1983-2000, 2001.

7. Cottrell GT, Lin R, Warn-Cramer BJ, Lau AF and Burt JM: Mechanism of $v$-Src-and mitogen-activated protein kinase-induced reduction of gap junction communication. Am J Physiol Cell Physiol 284: C511-C520, 2003.

8. Lichtenstein A, Minogue PJ, Beyer EC and Berthoud VM: Autophagy: a pathway that contributes to connexin degradation. J Cell Sci 124: 910-920, 2011.

9. Marmarou A, Foda MA, van den Brink W, Campbell J, Kita H and Demetriadou K: A new model of diffuse brain injury in rats. Part I: Pathophysiology and biomechanics. J Neurosurg 80: 291-300, 1994

10. Milad M, Vidal-Gonzalez I and Quirk G: Electrical stimulation of medial prefrontal cortex reduces conditioned fear in a temporally specific manner. Behav Neurosci 118: 389-394, 2004.

11. Song SX, Gao JL, Wang KJ, et al: Attenuation of brain edema and spatial learning deficits by the inhibition of NADPH oxidase activity using apocynin following diffuse traumatic brain injury in rats. Mol Med Rep 7: 327-331, 2013.

12. Meijer AJ and Codogno P: Autophagy: regulation and role in disease. Critical Rev Clin Lab Sci 46: 210-240, 2009.

13. Zhang YB, Li SX, Chen XP, et al: Autophagy is activated and might protect neurons from degeneration after traumatic brain injury. Neurosci Bull 24: 143-149, 2008.

14. Sun LQ, Gao JL, Cui CM, et al: Astrocytic p-connexin 43 regulates neuronal autophagy in the hippocampus following traumatic brain injury in rats. Mol Med Rep 9: 77-82, 2014

15. Luo CL, Li BX, Li QQ, et al: Autophagy is involved in traumatic brain injury-induced cell death and contributes to functional outcome deficits in mice. Neuroscience 184: 54-63, 2011.

16. Rami A, Volkmann T and Winckler J: Effective reduction of neuronal death by inhibiting gap junctional intercellular communication in a rodent model of global transient cerebral ischemia. Exp Neurol 170: 297-304, 2001.

17. Ohsumi A, Nawashiro H, Otani N, Ooigawa H, Toyooka T and Shima K: Temporal and spatial profile of phosphorylated connexin 43 after traumatic brain injury in rats. J Neurotrauma 27: 1255-1263, 2010.

18. Sasaki T and Garant PR: Fate of annular gap junctions in the papillary cells of the enamel organ in the rat incisor. Cell Tissue Res 246: 523-530, 1986.

19. Leach DH and Oliphant LW: Degradation of annular gap junctions of the equine hoof wall. Acta Anat (Basel) 120: 214-219, 1984.

20. Zhang YB, Li SX, Chen XP, et al: Autophagy is activated and might protect neurons from degeneration after traumatic brain injury. Neurosci Bull 24: 143-149, 2008. 